\title{
44.3: Photo-Aligned Passively Addressed Ferroelectric Liquid Crystal Display Cells: Effect of Aligning Layer Thickness
}

\author{
E. P. Pozhidaev, D. D. Huang, V. G. Chigrinov, Y. L. Ho, H. S. Kwok \\ The Hong Kong University of Science and Technology, Clear Water Bay, Kowloon, Hong Kong
}

\begin{abstract}
Effect of photoaligning azo-dye layers thickness on the alignment quality and multiplex operation of passively addressed ferroelectric liquid crystal (FLC) $1.5 \mu \mathrm{m}$ display cells has been investigated. An optimal (about 3 5 $\mathrm{nm}$ ) azo-dye layer thickness, that provides both the highest multiplex operation steadiness and the best contrast ratio of FLC display cells was found.
\end{abstract}

\section{Introduction}

A phenomenon of the bistability and multiplex mode degradation of passively addressed FLC display cells aligned with ordinary rubbing technique has been already discovered many years ago [1]. We are trying now to solve the problem using the photoalignment FLC technology, which enables to avoid mechanical brushing and looks therefore very promising [2]. Actually, photoanisotropic azo-dye films were used before for the alignment of FLC, but this approach does not prevent the bistability degradation [3]. At present approach we used a new water-soluble sulfonic azo-dye called SD-1 (Fig.1), which had been recently synthesized [4,5] and successfully tested for the alignment of ferroelectric liquid crystals [6].

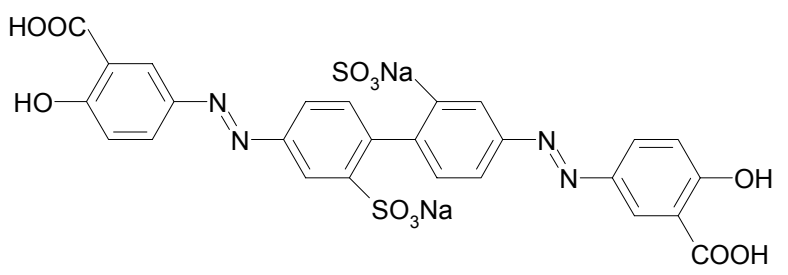

Figure 1. Chemical structure of SD-1 dye.

A remarkable property of this azo-dye is the pure reorientation of the molecular absorption oscillators perpendicular to the UV light polarization, which is practically not accompanied with photochemical transformations [6]. Probably, mentioned property provides a good chance to increase the chemical stability of photo aligning SD-1 layers and to prevent the bistability and multiplex mode degradation in this way. On the other hand, the uniformity and mechanical stability of any thin layer onto ITO surface depends on its thickness. This paper describes the effect of thickness of SD-1 aligning layers on the quality of FLC display cells operation.

\section{Experiments}

The best FLC alignment quality has been obtained for asymmetric boundary conditions [6]. At this approach only one ITO surface of FLC cells was prepared with SD-1 layer, but another one was simply washed in N,N-dimethylformamide (DMF) and covered with $1.5 \mu \mathrm{m}$ calibrated spacers. The various azo-dye SD-1 layer thickness onto ITO surface has been provided by different azodye concentrations in DMF solutions from $0.2 \%$ up to $1.3 \%$. The solutions were spin-coated onto ITO electrode at $3500 \mathrm{rpm}$, and dried at $100^{\circ} \mathrm{C}$. The UV light was irradiated onto surfaces of SD-1 layers during 1 hour using a super-high pressure $\mathrm{Hg}$ lamp, an interference filter at $365 \mathrm{~nm}$, and a polarizing filter. The light intensity on the surface of layer was $6 \mathrm{~mW} / \mathrm{cm}^{2}$ for the polarized light. The thickness of the azo-dye layer has been measured using the AFM method. The SD-1 film was formed on surfaces of test flat quartz plates, then a scratch line of about $10 \mu \mathrm{m}$ in width was produced using a tungsten tip. The surface with scratch line was scanned by AFM using a tapping mode (i.e. AFM tip is tapping onto the surface with a high frequency rather than contacting the surface continuously to prevent the damage of the film). The thickness was obtained from the cross-section analysis of the AFM scanning image. The film was discontinuous around dust particles with typical explosion regions of several micrometers, so one can measure the step of the film, and it agreed well with the scratch line method. The SD-1 layer thickness was varied from 0 to $12 \mathrm{~nm}$ dependent on the concentration of SD-1 in DMF as shown in Fig. 2.

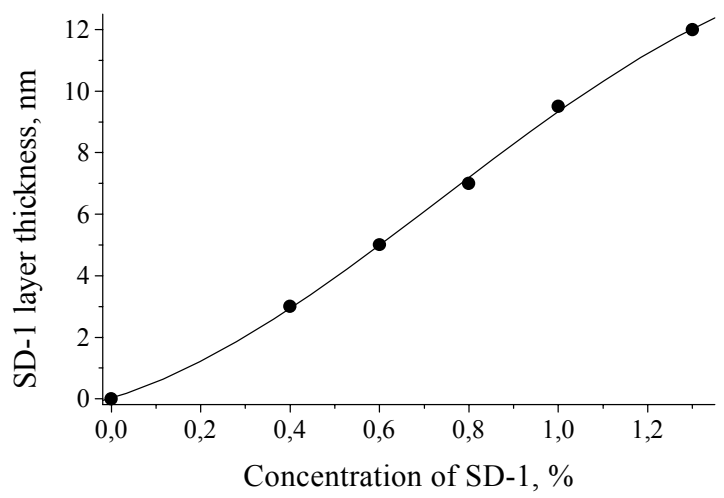

Figure 2. Dependence of SD-1 layer thickness on concentrations of SD-1 solutions in DMF spin-coated onto ITO surface at 3500 rpm.

Two glass substrates, one of them covered with SD-1 layer, while another one with $1.5 \mu \mathrm{m}$ spacers, were assembled to form FLC cell with a cell gap of $1.5 \mu \mathrm{m}$. The asymmetry of boundary conditions does not disturb the bistability, but minimizes the density of defects, because the solid surface covered with spacers provides the degenerated planar alignment, which occurs simply by washing it in DMF [6]. 
FLC mixtures FLC-408A and FLC-445 (from P. N. Lebedev Physical Institute of Russian Academy of Sciences) were injected into the cell in an isotropic phase by a capillary action. The FLC$408 \mathrm{~A}$ possesses the spontaneous polarization $P_{S}=150 \mathrm{nC} / \mathrm{cm}^{2}$ at $\mathrm{T}=23^{\circ} \mathrm{C}$, and the following phase transition sequence:

$$
\mathrm{Cr} \rightarrow{ }^{5^{\circ} \mathrm{C}} \rightarrow \mathrm{C}^{*} \rightarrow{ }^{54^{\circ} \mathrm{C}} \rightarrow \mathrm{A}^{*} \rightarrow{ }^{77^{\circ} \mathrm{C}} \rightarrow \mathrm{Is}
$$

The FLC-445 possesses the spontaneous polarization $P_{S}=210$ $\mathrm{nC} / \mathrm{cm}^{2}$ at $\mathrm{T}=23^{\circ} \mathrm{C}$, the phase transition sequence of this FLC writes:

$$
\mathrm{Cr} \rightarrow{ }^{6^{\circ} \mathrm{C}} \rightarrow \mathrm{C}^{*} \rightarrow{ }^{78^{\circ} \mathrm{C}} \rightarrow \mathrm{A}^{*} \rightarrow{ }^{90^{\circ} \mathrm{C}} \rightarrow \mathrm{Is}
$$

The helix pitch of both FLCs tends to infinity due to compensation in the bulk by two chiral dopants with the same signs of the spontaneous polarization and opposite signs of their handedness [7]. The electrooptical measurements have been carried out at $\lambda=0.6328 \mu \mathrm{m}$, with registration of responses with HP infinum oscilloscope and a photodiode.

\section{Results}

\subsection{Steadiness of multiplex operation}

All the cells prepared as described above manifested multiplex operation but the steadiness was strongly dependent on the driving voltage pulses duration and amplitude as well as on the thickness of the aligning layers. We propose a criterion of a multiplex mode steadiness as a ratio of noise $N$ in electrooptical response, produced by cross-talking pulses, to electrooptical signal $S$ generated by selecting pulses. Figure 3 illustrates a definition of the steadiness parameter $N / S$.

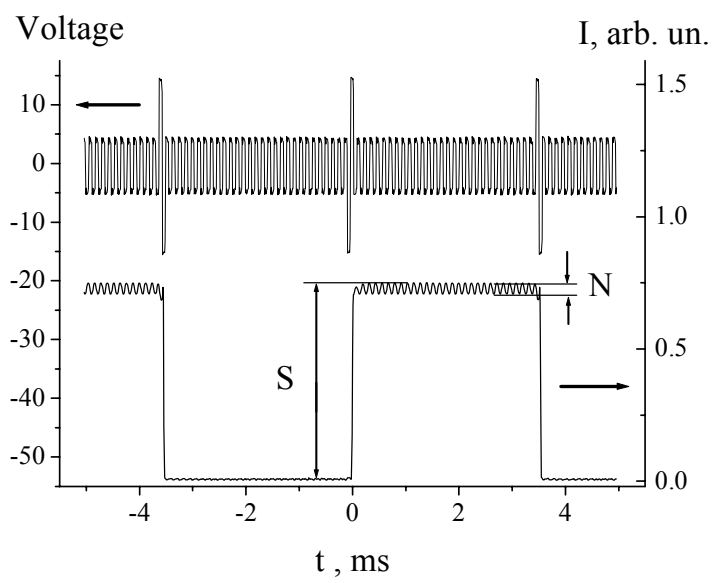

Figure 3. Typical multiplex driving voltage shape (top curve) and electrooptical response of the FLC-408A (bottom curve). The cell gap is $1.5 \mu \mathrm{m}$, the SD-1 layer thickness is $7 \mathrm{~nm}, \mathrm{~T}=25^{\circ} \mathrm{C}$.
The steadiness parameter varies $0 \leq N / S \leq 1$ and depends on driving voltage pulses duration as shown in Fig 4.

The dependence (Fig.4) could be explained as follows. If selecting pulses duration is too small for total FLC director switching, then no signal will appear and $N / S$ ratio tends to 1 . On the other hand, if the duration of cross-talking pulses is large enough for total FLC switching then a role of selecting pulses disappears and $N / S=1$ again. Minimal value of $N / S$ ratio, which can be close to zero just corresponds to the highest multiplex mode steadiness.

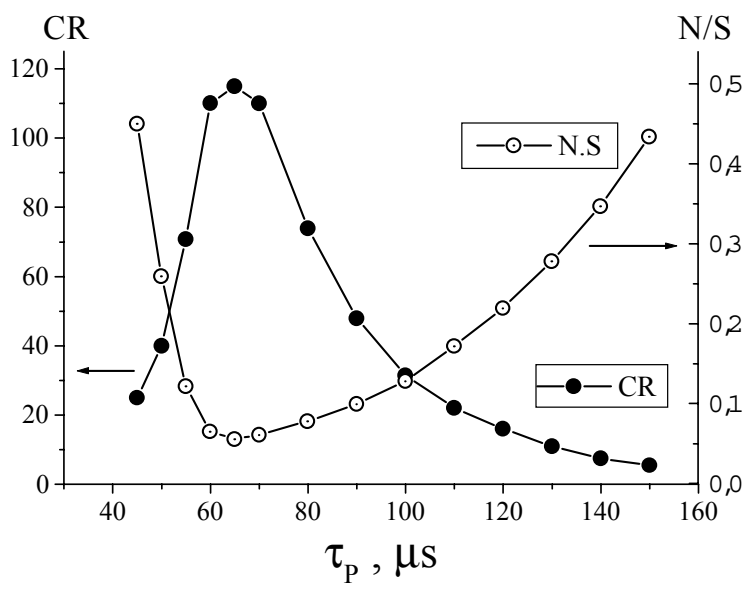

Figure 4. Dependence of $N / S$ parameter and the contrast ratio in multiplex mode on driving voltage pulses duration (the voltage has the same shape as in Fig. 3) for the $1.5 \mu \mathrm{m}$ FLC-408A cell, the SD-1 layer thickness is 3 $\mathrm{nm}, \mathrm{T}=25^{\circ} \mathrm{C}$.

A variation of the contrast ratio on driving voltage pulses duration $C R\left(\tau_{P}\right)$ has the same origin as already discussed $N / S\left(\tau_{P}\right)$ dependence, as principally defined by the noise from cross-talking pulses in the dark state of the electrooptical response. The contrast ratio characterizes the quality of FLC switching in a multiplex regime.

According to our measurements, minimum $(N / S)_{\min }$ of $N / S\left(\tau_{P}\right)$ as well as maximum $C R_{\max }$ of $C R\left(\tau_{P}\right)$ functions (Fig.4) depends both on FLC type and boundary conditions. The magnitude of $C R_{\max }$ strongly depends on the alignment quality. We need therefore to characterize the alignment quality and multiplex operation steadiness independently. The contrast ratio in bistable mode $C R_{b i s}$ (Fig.5) can be considered as a parameter of the alignment quality, while the value of noise to signal $(N / S)_{\min }$ (Fig.3) can illustrate the multiplex mode steadiness. Usually we have the contrast in multiplex operation lower, than the contrast in the bistable mode: $C R_{b i s} \gg C R_{\max }$

\subsection{Optimal thickness of aligning layers}

The dependence of contrast ratio $C R_{b i s}$ and $(N / S)_{\min }$ on SD-1 layer thickness $L_{D S-1}$ shows rather narrow region of the SD-1 layer thickness, appropriate for the operation of passively addressed photo-aligned FLC display cells. We can see (Fig.5), that the most steady multiplex operation of passively addressed FLC display cells as well as highest value of the contrast ratio is obtained, 
when the SD-1 azo-dye layer onto ITO surface is about $3 \sim 5 \mathrm{~nm}$ thick. The most suitable SD-1 layer thickness is about $3 \mathrm{~nm}$, which corresponds approximately to 5 6 mono-molecular layers of SD-1 planar aligned onto ITO surface.

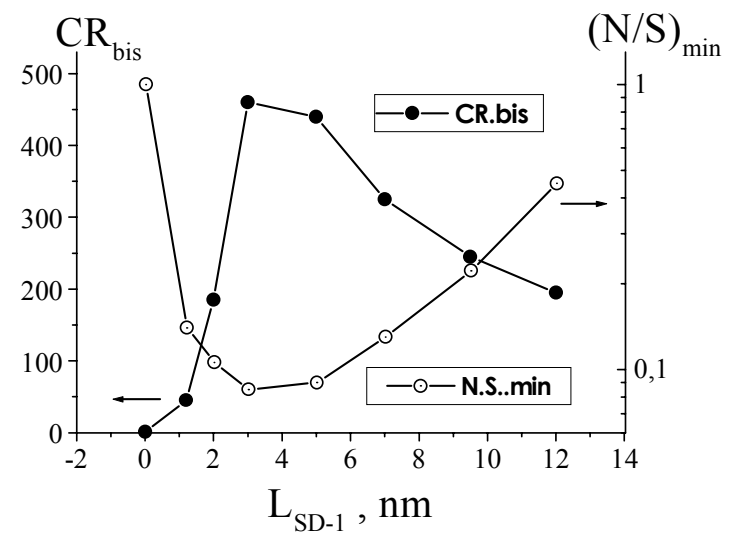

Figure 5. Dependence of the contrast ratio $C R_{b i s}$ and the steadiness parameter $(N / S)_{\min }$ of $1.5 \mu \mathrm{m}$ FLC-445 cells on the photo-aligning SD-1 layer thickness, $\mathrm{T}=25^{\circ} \mathrm{C}$.

A perfect multiplex mode occurs at optimal $3 \mathrm{~nm}$ aligning layer thickness (Fig.6) exhibiting $(N / S)_{\min }=0.05, C R_{\max }=120: 1$ while $C R_{b i s}=650: 1$, all measured at the wavelength of $\lambda=0.6328 \mu \mathrm{m}$.

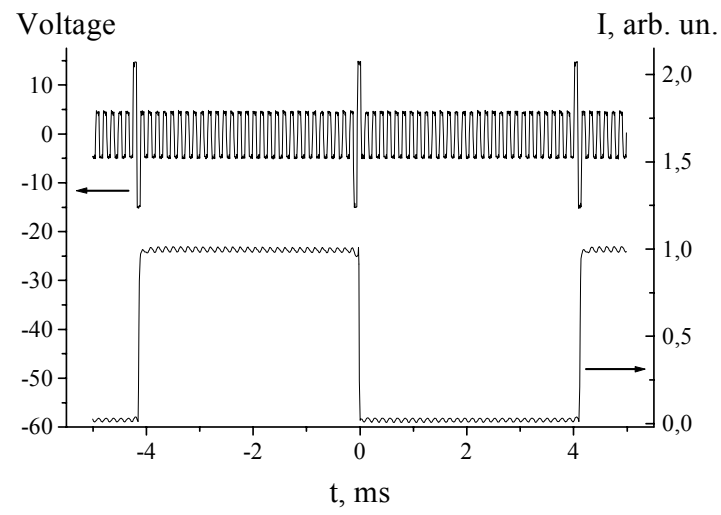

Figure 6. Typical multiplex driving voltage shape (top curve) and electrooptical response of the FLC-408A cell (bottom curve). The cell gap is $1.5 \mu \mathrm{m}$, the SD-1 layer thickness is $3 \mathrm{~nm}, \mathrm{~T}=25^{\circ} \mathrm{C}$.

The reason why the optimal aligning layer thickness is available for the multiplex mode is the highest uniformity of the FLC layer that occurs just at this optimal $3 \mathrm{~nm}$ SD-1 film thickness. The alignment textures of FLC layers evidently depend on the azo-dye layer thickness and rather poor aligning texture with irregular focal-conic domains may appear (Fig.7a). The domains occur due to the broken SD-1 layer, whose evaluated apparent thickness was about $1.2 \mathrm{~nm}$, i.e. approximately two SD-1 mono-layer are onto ITO surface. The SD-1 film discontinuity does not relate to dust particles in this case, but is an inherent property of the film. The explosion regions of discontinuous film are typically $10 \sim 100 \mu \mathrm{m}$. The texture in Fig. 7(b) illustrates the best FLC-445 alignment quality, when the thickness of SD-1 layer is about $3 \mathrm{~nm}$ of a completely continuous film. Only spacer defects of the texture are clearly visible here. This uniform texture is a consequence of the uniformity of the aligning layer. Increasing of the azo-dye layer thickness results in the appearance of regular striped domains possessing a periodicity $D$ along a normal to smectic layers of the FLC, see the photo in Fig. 7(c).
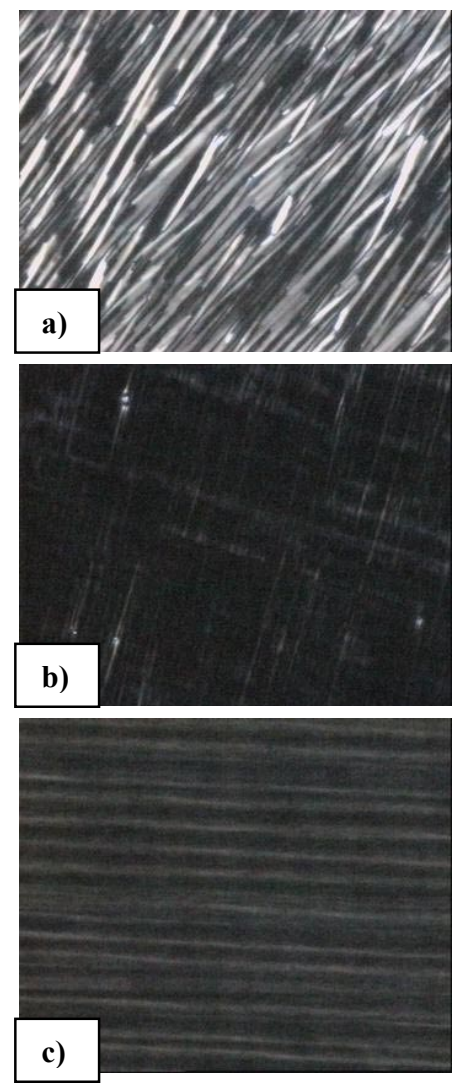

Figure 7. Textures of the 1,5 $\mu \mathrm{m}$ FLC-445 layers corresponding to different thickness $L_{S D-1}$ of the photo-aligning SD-1 layers: (a) $L_{S D-1} \cong 1.2 \mathrm{~nm}$, (b) $L_{S D-1} \cong 3 \mathrm{~nm}$, (c) $L_{S D-1} \cong 12 \mathrm{~nm}$. All images size is $117 \times 142 \mu \mathrm{m}^{2}$.

These domains are identified as ferroelectric domains in FLC cells [8], which create the modulation of the FLC apparent birefringence. The FLC layers become not perfectly uniform because of the domains appearance. Therefore the contrast ratio falls down, and the noise parameter $(N / S)_{\min }$ increases for $L_{S D-1}>3 \mathrm{~nm}$ (Fig.5). If SD-1 layer thickness grows further, the period of domains becomes larger, as shown in Fig. 8, and the domains become more distinct. 


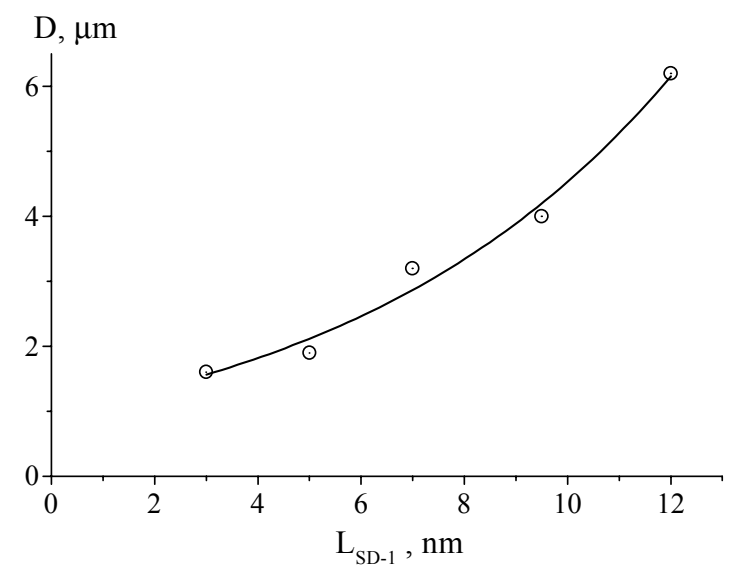

Figure 8. Dependence of ferroelectric domains periodicity in $1.5 \mu \mathrm{m}$ FLC-445 cells on thickness of the photoaligning SD-1 layer, $\mathrm{T}=25^{\circ} \mathrm{C}$.

\section{Discussion and Conclusion}

The optimal photo-aligning layers thickness is provided by a compromise between the two types of FLC layers instability: focal-conic domains and ferroelectric domains [8]. Focal-conic domains [9] are a consequence of aligning layers imperfection, they appear at very small aligning layer thickness. The ferroelectric domains [8] are an inherent property of FLC layers, however, the dependence of their periodicity on aligning layers thickness has never been reported so far. As a result ferroelectric domains become almost not visible at low thickness of the aligning layer, while focal conic domains are already suppressed in this case.

Very high quality of FLC's aligned by several mono-layers of SD1 azo-dye molecules results in a perfect multiplex mode operation of passively addressed FLC display cell with a contrast ratio of 120:1. The optimal uniform and unbroken $3 \sim 5 \mathrm{~nm}$ photo-aligning layers provide also high spatial optical uniformity of FLC cells, as it is shown in Fig. 9. So, photo-aligned FLC display cells exhibit remarkable properties that could be considered as a good scientific and technological background for the creation of passively addressed display devices.

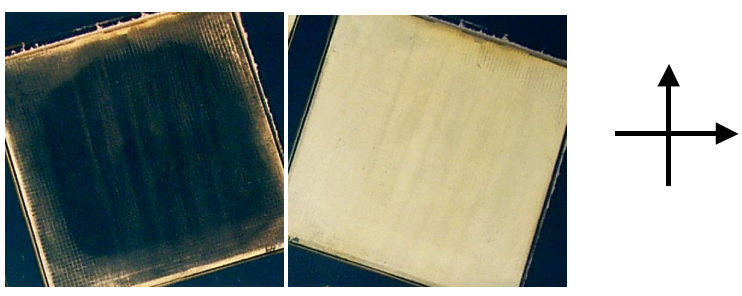

Figure 9. FLC-408A based 1,5 $\mu \mathrm{m}$ cell placed between the two crossed polarizers, shown by arrows. The light aperture of the cell is $33 \times 33 \mathrm{~mm}^{2}$. The dark and bright states are memorized after the driving voltage is switched off.

\section{Acknowledgements}

Financial support from RGC grant HKUST6004/01E is gratefully acknowledged.

\section{References}

[1] Y. Inaba, K.Katagiri, H. Inoue, J. Kanbe, S. Yoshihara, S. Iijuma, Ferroelectrics, 85, 255, (1988).

[2] M. Schadt, K. Schmitt, V. Kozenkov, V. Chigrinov, Jpn. J. Appl. Phys, 31, 2155, (1992).

[3] V. Vorflusev, V. Kozenkov, V. Chigrinov, Mol. Cryst. Liq. Cryst., 263, 577, (1995).

[4] W.C. Yip, E. K. Prudnikova, V.M. Kozenkov, V.G. Chigrinov, H.S. Kwok, H. Akiyama, M. Fukuda, H. Takada and H. Takatsu, SID'01 Digest, p. 1170 (2001).

[5] V. Chigrinov, E. Prudnikova, V. Kozenkov, Z. Ling, H. S. Kwok, H. Akiyama, T. Kawara, H. Takada, H. Takatsu, Liquid Crystals, 29, 1321, (2002).

[6] E. P. Pozhidaev, V. G. Chigrinov, D. D. Huang, H. S. Kwok, EURODISPLAY'2002 Digest, p.137, (2002).

[7] A. Z. Rabinovich, M.V. Loseva, N. I. Chernova, E. P. Pozhidaev, O. S. Petrashevich, J. S. Narkevich, Liquid Crystals, 6, 533, (1989).

[8] L.A. Beresnev, M.V. Loseva, N.I. Chernova, S.G. Kononov, P.V. Adomenas, E.P. Pozhidaev, Pisma v Jurn. Eksp. Teor. Fiziki (USSR), 51, 457, (1990).

[9] V.G. Chigrinov, Liquid Crystal Devices: Physics and Applications, Artech-House, Boston- London, 1999. 